\title{
Microbial Changes in the Rhizosphere of Paper Mulberry (Broussonetia papyrifera) Mutant with a High Concentration of Crude Protein
}

\author{
Zhaoxiang Wu ${ }^{1,2}$, Qiaoli Liu ${ }^{1,2}$, Yanqiang $\mathrm{Li}^{1,2}$, Huihu $\mathrm{Li}^{1,2}$, Yongda Zhong ${ }^{1,2}$, Faxin Yu ${ }^{1,2, ~ * ~}$ \\ ${ }^{1}$ Institute of Biological Resources, Jiangxi Academy of Sciences, Nanchang, China \\ ${ }^{2}$ The Key Laboratory of Horticultural Plant Genetic and Improvement of Jiangxi Province, Nanchang, China
}

Email address:

wuzhaoxiang@jxas.ac.cn (Zhaoxiang Wu),yufaxin@jxas.ac.cn (Faxin Yu)

*Corresponding author

To cite this article:

Zhaoxiang Wu, Qiaoli Liu, Yanqiang Li, Huihu Li, Yongda Zhong, Faxin Yu. Microbial Changes in the Rhizosphere of Paper Mulberry (Broussonetia papyrifera) Mutant with a High Concentration of Crude Protein. Earth Sciences. Vol. 10, No. 1, 2021, pp. 7-16. doi: 10.11648/j.earth.20211001.12

Received: February 2, 2021; Accepted: February 18, 2021; Published: February 26, 2021

\begin{abstract}
Recently, many studies involving plant-microorganism relationships in the rhizosphere of multitudinous important economic crops revealed a clear signature of the host plant in shaping its rhizosphere microbial composition and structure. The nutrient preference of host plant was suggested to be one important factor determining the structure and assembly of the rhizosphere microbiome, but the proof for this hypothesis is still not enough. In this study, soil microbiomes in the rhizosphere of two Paper mulberry varieties with different nitrogen absorption and utilization efficiency were investigated using a short term pot experiment in controlled greenhouse, and the physicochemical properties were also determined. The results showed that, compared to the control plants, the mutated Paper mulberry variety with high $\mathrm{N}$ demand reduced the microbial growth significantly and changed the bacterial and the fungal composition in the rhizosphere soils, and alkaline nitrogen was identified to be the most significant factor affecting soil microbial community. Moreover, the effects of excessive consumption of soil nutrient during Paper mulberry cultivation on the microbiome was revealed, and it could be employed in field water and fertilizer management of Paper mulberry planting. This study further confirmed that the soil nutrient status resulting from the plant nutrient preference drives the development of a plant-specific microbiome.
\end{abstract}

Keywords: Paper Mulberry, Nutrient Preference, MiSeq Sequencing, Rhizosphere, Soil Microbial Community

\section{Introduction}

The rhizosphere of plants, as the zone of interaction between plants' roots and microbes, plays an important role in the plant-microbe-soil system, facilitating host plant growth and development by the supply of various necessary nutrients [1], and also advancing activity and biodiversity of the microbial community through supporting organic carbon such as plant root exudates, mucilage, and sloughed-off root cells [2]. In the rhizosphere, the plant and the microbial community interacted with each other. Host plants influence soil microorganisms surrounding their roots by root morphology, excretion of exudates, and other substances that can be both repellent and chemoattractant signals, and the presence of dead root cells [3-4]. Conversely, the soil microorganisms colonizing in the rhizosphere affect the host plants through regulation of plant growth hormone [5], activation of soil nutrients [6], and degradation of toxic and harmful substances by synthesis and secretion of secondary metabolites [7], and long-distance transport of water and nutrients [8]. The plant-associated microbial community in the rhizosphere also referred to as the second genome of the plant, is crucial for plant growth and health [9-10].

Among various factors influencing the soil microbial composition in the rhizosphere of the host plants, plant species have been recognized as the best predictor of the composition of fungal and bacterial communities [11]. However, this microbial community is also influenced by the physicochemical properties of the soil [12]. Recent advances in plant-microbe interactions have revealed a clear signature 
of the host plant in shaping its rhizosphere microbiome, as evidenced by the specific microbial communities hosted by different plant species growing in the same soil [13-14]. However, whether these host-specific communities thriving in the rhizosphere are directly recruited by plant-derived carbons (root exudates) or via preferences for specific soil conditions, such as mineral nutrients and physical structure, is still unknown. The research by Ai et al. [14] indicated that a limited capability of root-derived products to influence the rhizosphere microbiota. Thus, considering the specificity of plant species with respect to the level and types of soil nutrients absorbed, plant nutrient preferences may also play a key role in recruiting the host-dependent rhizosphere microbiome.

Paper mulberry (Broussonetia papyrifera) is an ecologically, economically, and medicinally important plant with variety of features such as fast growth, ease of breeding, strong tillering ability, and pruning resistance [15]. Belonging to the nitrogen-fixing clade of Rosales and Moraceae, Paper mulberry might fix atmospheric nitrogen with formation of nodules by symbiotic microbes and transformed it into protein stored in leaf [16]. More recently, Paper mulberry has also been used as forage to address the shortage of feedstuff because of its digestible crude fiber and high protein contents [17-18]. To further improve forage quality of Paper mulberry, various efforts were taken in several areas to increase crude protein content, including breeding new varieties and improving planting technology. A new hybrid of Paper mulberry with a high crude protein concentration has been cultivated by Shen and Peng [19] and planted in almost all provinces of China as part of a national project to resolve the silage deficiency. Based on tissue culture of the hybrid material, we further obtained a new mutated variety of Paper mulberry (named as FT temporarily) with higher crude protein concentration taking advantage of accelerated heavy ion irradiation. Nitrogen was quickly absorbed, transported, and stored in different tissues by the FT plants, which led to serious nitrogen deficiency in the soil if the soil nitrogen pool can not be supplemented in time. How nitrogen preference of the FT plants affected soil physicochemical properties and the microbial community was still unknown.

Due to the great consequence of plant-microorganism relations in the rhizosphere soil for nutrient cycling and plant growth and health, it is essential to recognize factors affecting microbial parameters in the soil environment. And the role of plant nutrient preferences in recruiting the host-dependent rhizosphere microbiome was speculated. Therefore, to verify this hypothesis, the rhizosphere microbiomes of two varieties of Paper mulberry with different nitrogen absorption and utilization efficiency were determined using high-throughput MiSeq sequencing as well as the soil nutrient properties in a monocropping system with a short term pot experiment in control. The aims of this study were (1) to determine whether nitrogen nutrient preference is involved in assembling a host-specific rhizosphere microbiome and (2) to elucidate how Paper mulberry nitrogen nutrient preference shapes the rhizosphere microbiome.

\section{Materials and Methods}

\subsection{Plant Materials and Growth Conditions}

The hybrid paper mulberry does not produce flowers, so the first-generation vegetative propagators were used for the following experimental materials. Regenerated plantlets (wild type $(\mathrm{CK})$ and the mutant with a high concentration of crude protein (FT) cultivated by accelerated $12 \mathrm{C}^{6+}$ irradiation, $\sim 5$ $\mathrm{cm}$ in height) were prepared and transferred to $300-\mathrm{ml}$ plastic pots (one plant per pot) into a mixture (1:1, by vol.) of peat soil: sand. The mixture had $\mathrm{pH} 4.32, \mathrm{EC} 16.43 \mathrm{dS} / \mathrm{m}$, organic carbon $38.02 \mathrm{~g} / \mathrm{kg}$ and $340.2 \mathrm{mg} / \mathrm{kg}$ available $\mathrm{N}$ (alkaline $\mathrm{KMnO}_{4}$ extractable), $223.48 \mathrm{mg} / \mathrm{kg}$ Olsen-P $\left(\mathrm{NH}_{4} \mathrm{~F}-\mathrm{HCl}\right.$ extractable) and $21.86 \mathrm{mg} / \mathrm{kg}$ available $\mathrm{K}$ (1 $\mathrm{M} \mathrm{NH}_{4} \mathrm{OAc}$ extractable).

Paper mulberry plants were grown in a random design in the climate-controlled chamber $(16 \mathrm{~h}$ photoperiod at a light density of approximately $400 \mu \mathrm{mol} \cdot \mathrm{m}^{-2} \cdot \mathrm{s}^{-1}$ photosynthetically active radiation, $23-25^{\circ} \mathrm{C}$ and $60 \%$ relative humidity) at the Jiangxi academy of sciences for 45 days ( 4 plants per variety) and watered with tap water once a week.

\subsection{Harvesting and Sampling}

At harvest, rhizosphere soil was carefully collected from fine roots by gently scraping adhering soil using fine forceps. The soil samples were passed through $2 \mathrm{~mm}$ mesh to remove plant debris, thoroughly homogenized and divided into subsamples. Subsamples for molecular analysis were frozen stored at $-80^{\circ} \mathrm{C}$, and subsamples for analysis of soil physicochemical properties were air-dried.

Leaf, stem, and root were separated, washed, and gently dried with paper towels before being weighed. Total fresh weights of tissues were determined, and a weighed leaf sample (100-200 mg) was taken for determination of Chlorophyll concentration. Plant tissues were dried at $\sim 45^{\circ} \mathrm{C}$ for $3-4$ days before dry weight was determined.

\subsection{Plant Growth and Soil Physicochemical Characteristics Analysis}

The total leaf dry weight was determined from the total fresh weight and the fresh/dry weight ratio of the sample. Excised leaflets were collected in plastic bags and were weighed within one hour of sampling and extracted by $100 \%$ dimethyl sulfoxide for $24 \mathrm{~h}$ in the dark, to determine the content of Chla, Chlb, total chlorophylls as described previously [20]. Dried plant tissues were ground, and stem and leaf were mixed together as shoot samples. Crude protein concentration was determined by the Kjeldahl method.

Soil $\mathrm{pH}$ was determined using glass electrode $\mathrm{pH}$ meter (FE20-Five Easy Plus ${ }^{\mathrm{TM}}$, Switzerland) in a 1: 2.5 soil/water $(\mathrm{w} / \mathrm{v})$ suspension [21], and electrical conductivity (EC) was measured for the water extracts containing the 1:5 soil/water ratios (w/v). Soil organic carbon (SOC) was determined by the 
$\mathrm{K}_{2} \mathrm{Cr}_{2} \mathrm{O}_{7}$ oxidation oxidation-reduction titration method [22]. Soil inorganic nitrogen was assayed by using the alkaline hydrolysis method [23] and soil available phosphorus (P) by Mo-Sb colorimetry method [24]. Microbial Biomass C in Soils was determined by the Chloroform Fumigation-Extraction Method.

\subsection{Soil DNA Extraction and Sequencing}

Soil DNA was extracted from $0.5 \mathrm{~g}$ subsample of each soil sample using the Fast DNA ${ }^{\circledR}$ SPIN Kit for Soil (MP Biomedicals, Solon, Ohio, USA). The extracted total DNA, dissolved in $30 \mu \mathrm{l}$ sterilized deionized water, was checked on a $1 \%$ agarose gel, and the DNA concentration and quality were estimated using a Nanodrop ${ }^{\circledR}$ ND-2000 UV-Vis Spectrophotometer (NanoDrop Technologies, USA). Amplicon libraries for bacteria were produced by using bacteria-specific primers $515 \mathrm{~F}$ and $806 \mathrm{R}$ designed for the V4 region of the $16 \mathrm{~S}$ rRNA gene according to Bergmann et al. [25]. Each pair of primers used to amplify a certain sample was barcoded with a unique error-correcting 10-12 bases barcode on both forward and reverse primers. All amplifications were performed in 2 -fold $25 \mu 1$ reactions, while each PCR reaction was carried out in $25 \mu$ l volume, including $1 \mu \mathrm{l}$ of template DNA $(20 \mathrm{ng} / \mu \mathrm{l}), 1 \mu \mathrm{l}$ of each primer $(10 \mathrm{pmol}$ $\mu \mathrm{l}^{-1}$ ), $12.5 \mu \mathrm{l}$ of $2 \times$ PCR reaction mix (TAKARA Biotechnology Co. Ltd), and $0.5 \mu$ of Taq DNA polymerase

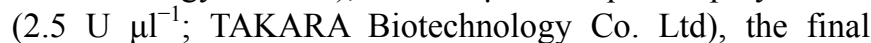
volume was added up to $25 \mu \mathrm{l}$ with sterilized deionized water. The protocol of amplification was as follows: $5 \mathrm{~min}$ initial denaturation at $95^{\circ} \mathrm{C}$, followed by 35 cycles of denaturation for $45 \mathrm{~s}$ at $94^{\circ} \mathrm{C}, 45 \mathrm{~s}$ primer annealing at $58^{\circ} \mathrm{C}, 1 \mathrm{~min}$ extension at $72^{\circ} \mathrm{C}$, and a final $10 \mathrm{~min}$ extension at $72^{\circ} \mathrm{C}$. Amplicon libraries for fungi were prepared using an identical approach, except that we chose a specific primer pair ITS1F and ITS2 [26], designed for the internal transcribed spacer (ITS) 1 region for Illumina sequencing. PCR products of each sample were pooled and purified by agarose gel electrophoresis with a QIAquick PCR Purification Kit (QIAEX II Gel Extraction Kit, QIAGEN Sciences, Maryland), estimated by using a Nanodrop ${ }^{\mathbb{R}}$ ND-2000 UV-Vis Spectrophotometer (NanoDrop Technologies, USA) and mixed together with an equal amount of $150 \mathrm{ng}$ for each sample. The pooled samples were sent to Shanghai Majorbio Bio-Pharm Technology Co., Ltd, China, and sequenced on an Illumina Miseq2000 platform (Illumina, San Diego, CA, U.S.A.).

\subsection{Bioinformatics and Statistical Analysis}

Data yielded from Illumina sequencing was analyzed with the Quantitative Insights Into Microbial Ecology (QIIME) pipeline (v.1.9.1), as described previously by Caporaso et al. [27]. All the raw sequence obtained was assigned to each sample based on their specific barcode sequence. Reads with an average quality value of $<30$ and not having both universal primer sequences were all filtered off. For bacteria, following quality control, removal of chimeras and nontargeted sequences, the left sequences were aligned with the Silva-ARB database (version 119) [28], and clustered into operational taxonomic units (OTUs) at $97 \%$ identity by using the unsupervised Bayesian clustering algorithm CROP [29]. For fungi, data were analyzed with an identical procedure except that ITS1 region was extracted from each sequence by Fungal ITS Extractor 1.1 after removal of primers [30], and sequences were aligned against the UNITE ITS database (version 6.0) [31]. To estimate the final ranking, the abundance-based coverage estimator (ACE), the estimated asymptotic microbial taxon richness (Chao), and the observed richness (sobs) were calculated. After an identical number of reads (43 804 and 49172 for bacteria and fungi respectively) subsampled from each sample, microbial diversity was compared between varieties based on the calculated Shannon index. Finally, sequences were assigned to taxonomic groups by using the RDP classifier (Rel. 11).

All microbial community-related analyses were based on the relative abundance of OTUs per sample unless otherwise stated, and the taxonomic community dissimilarity was calculated by using the Bray-Curtis index among varieties. Principal Co-ordinates Analysis (PCoA) of microbial communities was carried out by using the APE package in R [32]. Taxonomic community composition was compared between the mutant and CK by using ANOSIM [33]. Differences in observed parameters between the mutant and CK were tested by one-way ANOVA with the software package SPSS 18.0 (SPSS Inc., Chicago, IL, USA). The phylogenetic trees were constructed by using the Maximum Likelihood method based on the Kimura 2-parameter model, with the nucleotide sequences of the 30 most abundant species.

To investigate molecular-level functional traits, a PICRUSt (https://picrust.github.com) was performed for the bacterial community [34]. 16S rRNA gene abundance levels were normalized against the known gene copy number and function predictions were performed based on the OTU table with PICRUSt. Functional predictions were categorized into enzyme and statistical analysis was performed using the SPSS 18.0 software package (SPSS 18.0; SPSS, Chicago, IL, USA).

\section{Results}

\subsection{Plant Growth and Soil Physicochemical Fluctuation}

The results showed that FT exhibited fast growth, and had a higher dry matter and nutrient accumulation. In terms of biomass, leaf dry weight increased $24.07 \%$ in FT, stem increased $74.68 \%$ and root increased $74.62 \%$ (Table 1). Similar to biomass, chlorophyll a, chlorophyll b and total chlorophyll concentration were higher in FT than CK (the control plant) $(P<0.05)$. As the main nutritional component of animal feed, crude protein becomes the most concerned objective of feed crop breeders. Crude protein concentration in FT was found to be higher than that in the control, increasing by $12.68 \%$ in shoot and $25.53 \%$ in the root.

The growth of paper mulberry greatly consumed soil 
nutrients and reduced soil $\mathrm{pH}$ and electrical conductivity. Both for FT and CK, the soil physicochemical characteristics were much lower than that before paper mulberry cultivation. However, except for $\mathrm{pH}$ and Olsen-P, the soil physicochemical characteristics for FT were lower than those for CK (Table 2, $P<0.05)$. Notably, soil microbial C was lower for FT than that for CK (Figure 1), with a similar pattern of the soil physicochemical characteristics.

Table 1. Plant growth, Chlorophyll concentration, and matter accumulation between the two varieties of Paper mulberry.

\begin{tabular}{llllllll}
\hline & Biomass (g) & & & \multicolumn{2}{c}{ Chlorophyll (mg/g) } & \multicolumn{2}{c}{ Crude protein (mg/g) } \\
\hline & Leaf & Stem & Root & a & b & a+b & Shoot \\
\hline CK & 2.41 & 0.79 & 1.30 & 1.17 & 0.24 & 1.41 & 160.44 \\
FT & 2.99 & 1.38 & 2.27 & 1.28 & 0.34 & 1.63 & 180.78 \\
Sig & $*$ & $*$ & $* *$ & $*$ & $* * *$ & $* *$ & $*$ \\
\hline
\end{tabular}

Notes: Sig., significance; ${ }^{*}, P<0.05 ; * *, P<0.01 ; * * *, P<0.001$.

Table 2. The physicochemical properties of the mixtures after paper mulberry cultivation.

\begin{tabular}{llllll}
\hline & $\mathbf{p H}$ & Electrical conductivity & $(\mathbf{m S} / \mathbf{c m})$ & Organic carbon $(\mathbf{g} / \mathbf{k g})$ & Alkaline nitrogen $(\mathbf{m g} / \mathbf{k g})$ \\
\hline CK & $3.85 \pm 0.02$ & $15.51 \pm 0.10$ & $37.57 \pm 1.42$ & $105.03 \pm 2.50$ \\
FT & $3.76 \pm 0.04$ & $14.57 \pm 0.11$ & $32.36 \pm 1.16$ & $76.30 \pm 8.47$ \\
Sig. & ns & $* *$ & $*$ & $*$ & $168.20 \pm 13.34$ \\
\hline
\end{tabular}

Notes: Sig., significance; *, $P<0.05 ; * *, P<0.01$; ***, $P<0.001$.

\subsection{Sequencing Results and Microbial Diversity Indices}

After quality control, removal of chimeras and nontargeted sequences, 351986 bacterial sequences (ranging from 43 804 69 289 sequences per sample), and 380943 fungal sequences (ranging from 49 172 73 122 sequences per sample) were used for further analysis. A total of 1996 bacterial operational taxonomic units (OTUs) and 1924 fungal OTUs were identified in rhizosphere soils of Paper mulberry at a cut-off of $97 \%$ sequence similarity. The fungal OTUs detected in rhizosphere soil were more abundant in CK than FT $(P=$ $0.022)$, but the difference was not significant for bacteria $(P=$ 0.225 ). Rarefaction waves showed that the number of OTUs increased with the number of sequences obtained in each of the soil samples, and the curves already reached a plateau at $97 \%$ similarity level (Figure 2), suggesting that the sequencing was deep enough to cover the microbial community in the rhizosphere soil of Paper mulberry.

For bacteria, Chao 1 at the dissimilarity of 0.03 was higher in the rhizosphere soil of CK than FT, but the Shannon index and ACE showed no significant difference. However, the Shannon index, ACE and Chaol were all higher in the rhizosphere soil of CK than FT for the fungal community (Table 3). Overall, the fungal community alpha diversity was significantly lower in the rhizosphere soils of FT, but the difference for bacteria was not significant. Bacterial community dissimilarity was significantly lower in the rhizosphere soils of FT (Figure 3C, $P$ $=0.042)$, and the fungal community dissimilarity showed the same trend (Figure 4C, $P=0.029$ ), which indicated that both bacterial and fungal community in rhizosphere soils of FT were more similar in composition.

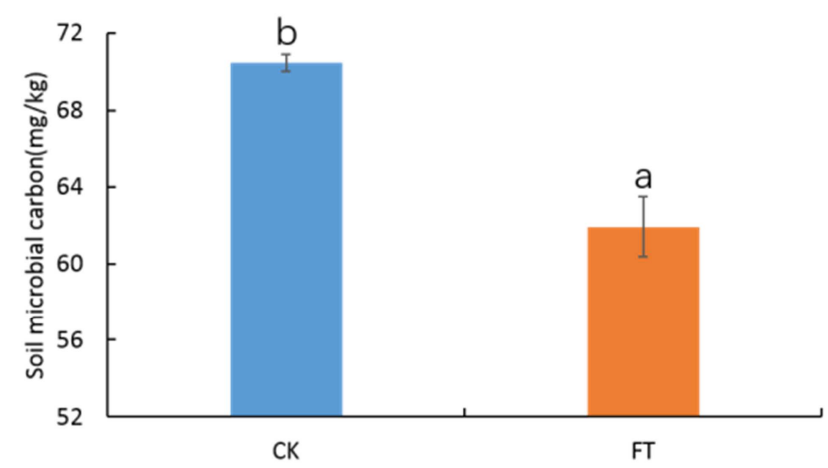

Figure 1. Soil microbial carbon concentration after paper mulberry cultivation.
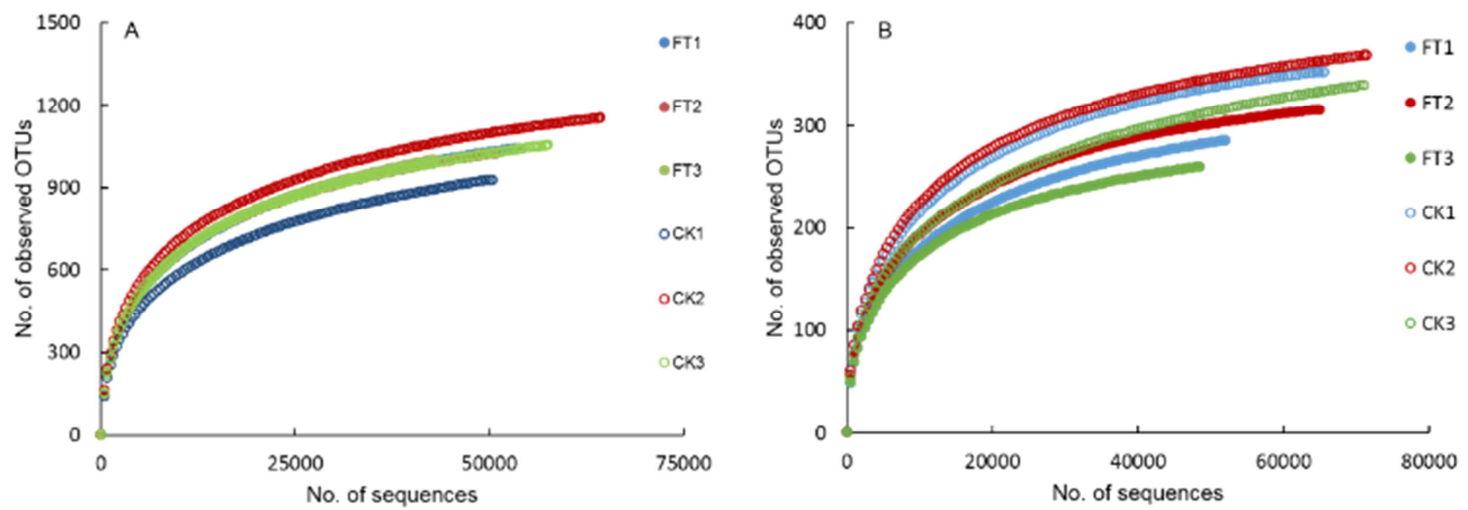

Figure 2. Rarefaction curves of bacteria $(A)$ and fungi $(B)$ associated with the rhizospheric soil of Paper mulberry depicting the effects of $3 \%$ dissimilarity on the number of OTUs identified. Hollow circles, the control; Solid circles, mutant with high protein concentration. 
Table 3. Microbial diversity indexes in the rhizosphere soils of Paper mulberry.

\begin{tabular}{llllll}
\hline & & OTUs & Shannon & Ace & Chao1 \\
\hline \multirow{2}{*}{ Bacteria } & CK & $343.33 \pm 13.57$ & $4.16 \pm 0.10$ & $376.60 \pm 10.54$ & $379.87 \pm 11.18^{*}$ \\
& FT & $322.00 \pm 6.08$ & $4.23 \pm 0.01$ & $351.72 \pm 3.45$ & $346.58 \pm 5.59$ \\
Fungi & CK & $354.00 \pm 8.39^{*}$ & $3.05 \pm 0.11^{*}$ & $395.99 \pm 6.82^{*}$ & $385.59 \pm 6.13^{*}$ \\
& FT & $287.33 \pm 16.18$ & $2.76 \pm 0.13$ & $322.29 \pm 17.94$ & $325.47 \pm 19.74$ \\
\hline
\end{tabular}

Notes: $*, P<0.05$.

\subsection{Microbial Community in the Rhizosphere of the Paper Mulberry}

PCoA based on the relative abundance of OTUs separated microbial community in the rhizosphere soils of FT from $\mathrm{CK}$ at the first axis (Figure 3A and Figure 4A). The hierarchical clustering tree on the OTU level further strengthened the difference of microbial community between FT and the control plants (Figure 3B and Figure 4B). By classifying all OTUs into taxonomic groups, we identified 20 bacterial phyla and 7 fungal phyla associated with rhizosphere soils (Table 4). The dominant

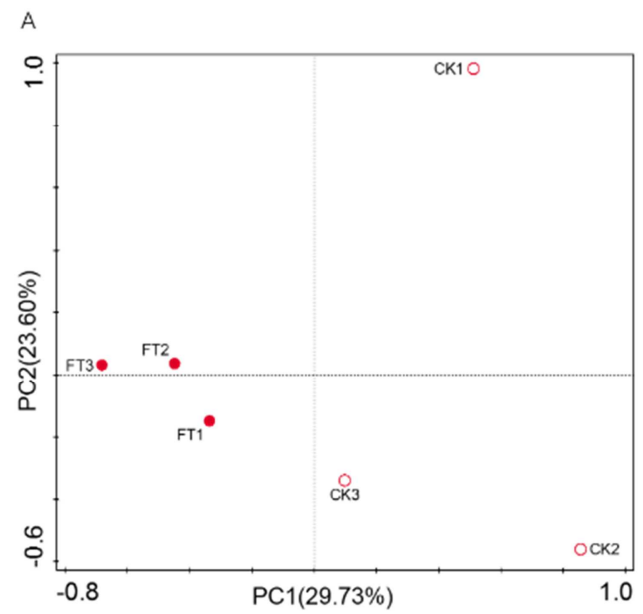

bacterial phyla in both FT and CK were Actinobacteria, Proteobacteria, Chloroflexi, Patescibacteria, Bacteroidetes, Acidobacteria, and Planctomycetes. By the T-test, Gemmatimonadetes and Patescibacteria were found to be less abundant $(P=0.02,0.006$, respectively), but Acidobacteria more abundant in FT, compared to the control plants $(P=0.026)$. On the other side, the dominant fungal phyla were Ascomycota, Basidiomycota, and Mortierellomycota. Basidiomycota and Rozellomycota were more abundant in FT than the control plants $(P=0.046,0.011$, respectively).

B

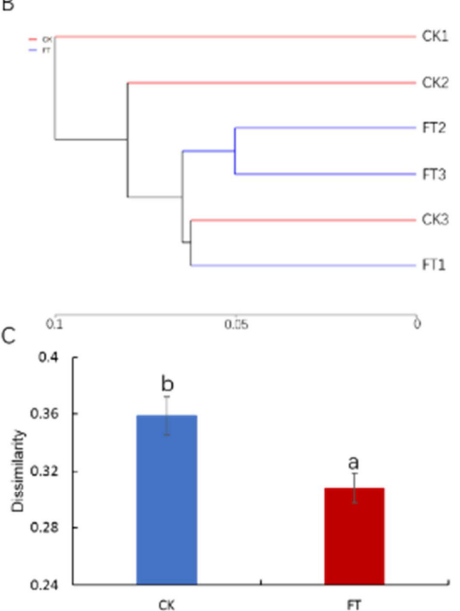

Figure 3. The structure of microbial community for bacteria associated with rhizospheric soil of Paper mulberry. A, Principal coordinate analyse (PCoA) on OTU level; B, Hierarchical clustering on OTU level; $C$, Comparison on average taxonomic dissimilarity (Bray-Curtis) of bacterial communities.

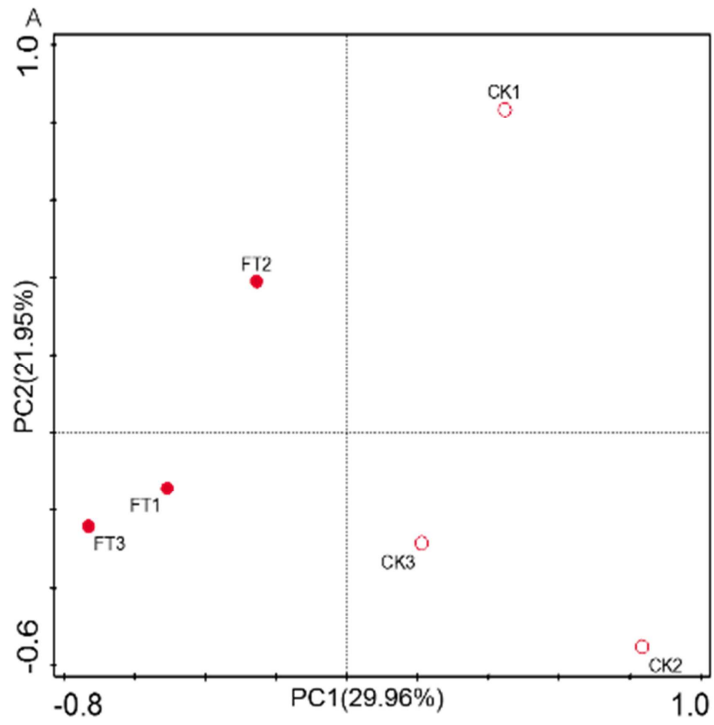

B
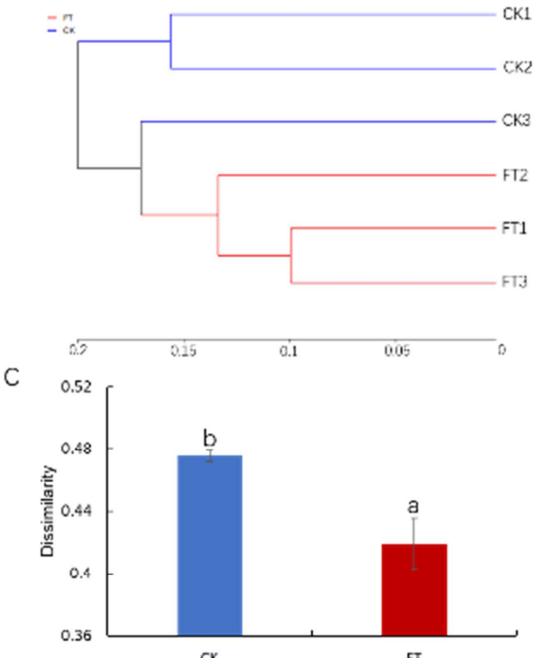

Figure 4. The structure of microbial community for fungi associated with rhizospheric soil of Paper mulberry. A, Principal coordinate analyse (PCoA) on OTU level; B, Hierarchical clustering on OTU level; $C$, Comparison on average taxonomic dissimilarity (Bray-Curtis) of bacterial communities. 
Table 4. Microbial compositions of FT and the control plant at the phylum level. Datas show the average percentage of phylum proportion in the rhizosphere soil of paper mulberry.

\begin{tabular}{|c|c|c|c|c|}
\hline & \multirow{2}{*}{ Phylum } & \multicolumn{3}{|c|}{ Rhizosphere soil (\%) } \\
\hline & & FT & CK & Sig. \\
\hline \multirow{21}{*}{ Bacteria } & Thaumarchaeota & 0.12460 & 0.12774 & $\mathrm{NS}$ \\
\hline & FBP & 0.06191 & 0.05564 & NS \\
\hline & WPS-2 & 0.13871 & 0.09874 & NS \\
\hline & BRC1 & 0 & 0.00392 & NS \\
\hline & Firmicutes & 1.02504 & 0.62458 & NS \\
\hline & Entotheonellaeota & 0 & 0.00157 & NS \\
\hline & Cyanobacteria & 0.47177 & 0.36597 & NS \\
\hline & Rokubacteria & 0 & 0.00313 & NS \\
\hline & Actinobacteria & 46.03425 & 34.26903 & NS \\
\hline & Verrucomicrobia & 0.14184 & 0.11755 & NS \\
\hline & Dependentiae & 0.15987 & 0.16771 & NS \\
\hline & Gemmatimonadetes & $0.65436 \downarrow$ & 1.02817 & $*$ \\
\hline & Bacteroidetes & 1.63473 & 4.22162 & NS \\
\hline & Acidobacteria & $2.52576 \uparrow$ & 1.80792 & $*$ \\
\hline & Planctomycetes & 1.44744 & 2.22248 & NS \\
\hline & Chloroflexi & 9.60699 & 6.76306 & NS \\
\hline & Proteobacteria & 31.90392 & 40.68101 & NS \\
\hline & Nitrospirae & 0.02429 & 0.02429 & NS \\
\hline & Armatimonadetes & 0.02508 & 0.02038 & NS \\
\hline & Patescibacteria & $3.47087 \downarrow$ & 6.99894 & $* *$ \\
\hline & unclassified Bacteria & 0.54857 & 0.39654 & NS \\
\hline \multirow{8}{*}{ Fungi } & Ascomycota & 76.39375 & 79.91581 & NS \\
\hline & Basidiomycota & $12.82545 \uparrow$ & 7.43539 & $*$ \\
\hline & Chytridiomycota & 0.03588 & 0.73008 & NS \\
\hline & Entorrhizomycota & 0.00138 & 0 & NS \\
\hline & GS19 & 0.00138 & 0 & NS \\
\hline & Mortierellomycota & 0.24980 & 0.24290 & NS \\
\hline & Rozellomycota & $0.00759 \uparrow$ & 0.00069 & $*$ \\
\hline & unclassified Fungi & 10.48477 & 11.67512 & NS \\
\hline
\end{tabular}

Note: FT, the mutant plants; CK, the control plants; Sig., significance; ${ }^{*}, P$ $<0.01$; * $P<0.05$; NS, $P>0.05$.

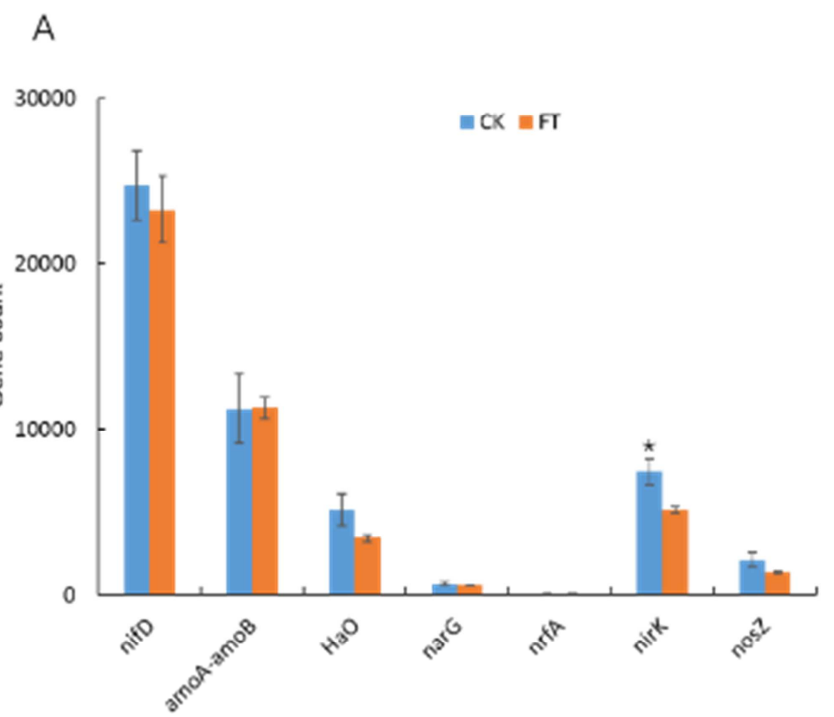

The 30 most abundant genus of the bacterial and the fungal community was selected to construct the phylogenetic tree respectively, and the abundance of the selected genus was compared between FT and CK. The results showed that the 30 most abundant bacterial genus belonged to just 5 phyla (Bacteroidetes, Actinobacteria, Proteobacteria, Patescibacteria, and Chloroflexi), and Streptomyces was found to be more abundant $(P=0.002)$, but norank_Chitinophagaceae less abundant in FT compared to the control plants $(P=0.02)$ (Figure 5). On the other side, the 30 most abundant fungal genus belonged to 4 phyla (Ascomycota, Mortierellomycota, Basidiomycota, and Chytridiomycota), and Trichoderma, Fusarium, Cladophialophora, and Knufia were all found to be less abundant in FT $(P=0.021,0.003,0.049$, and $P<0.001$, respectively, Figure 6 ).

To further study the effects of environmental factors on the microbial community, Pearson correlation analysis was performed between soil physicochemical characteristics and microbial composition at the genus level. Alkaline nitrogen was found to be the most significant factor affecting soil microbial community, followed by electrical conductivity, organic carbon, and $\mathrm{pH}$, but Olsen-P showed no significant effect. Alkaline nitrogen was found to negatively correlate with Streptomyces $(P=0.015)$, positive with Trichoderma, Fusarium, Cladophialophora, and Knufia $(P=0.015,0.025$, 0.036 and 0.015 , respectively, Table 5). Streptomyces exhibited to negatively correlate with most of the soil physicochemical characteristics, and Cladophialophora showed the opposite trend.

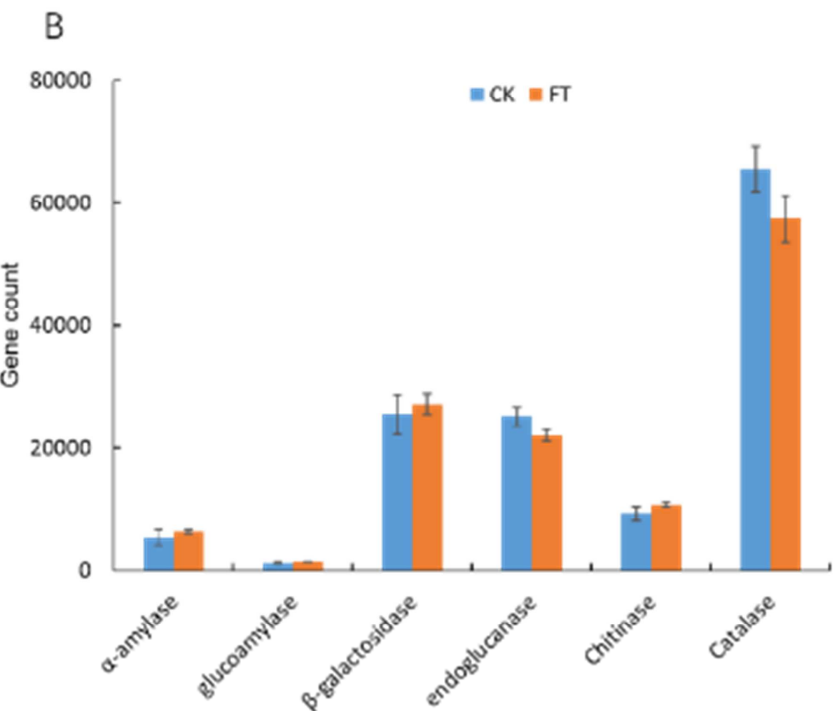

Figure 5. Phylogenetic tree on the 30 most abundant genus for bacterial community. ${ }^{*}, P<0.05 ; * *, P<0.01$. 


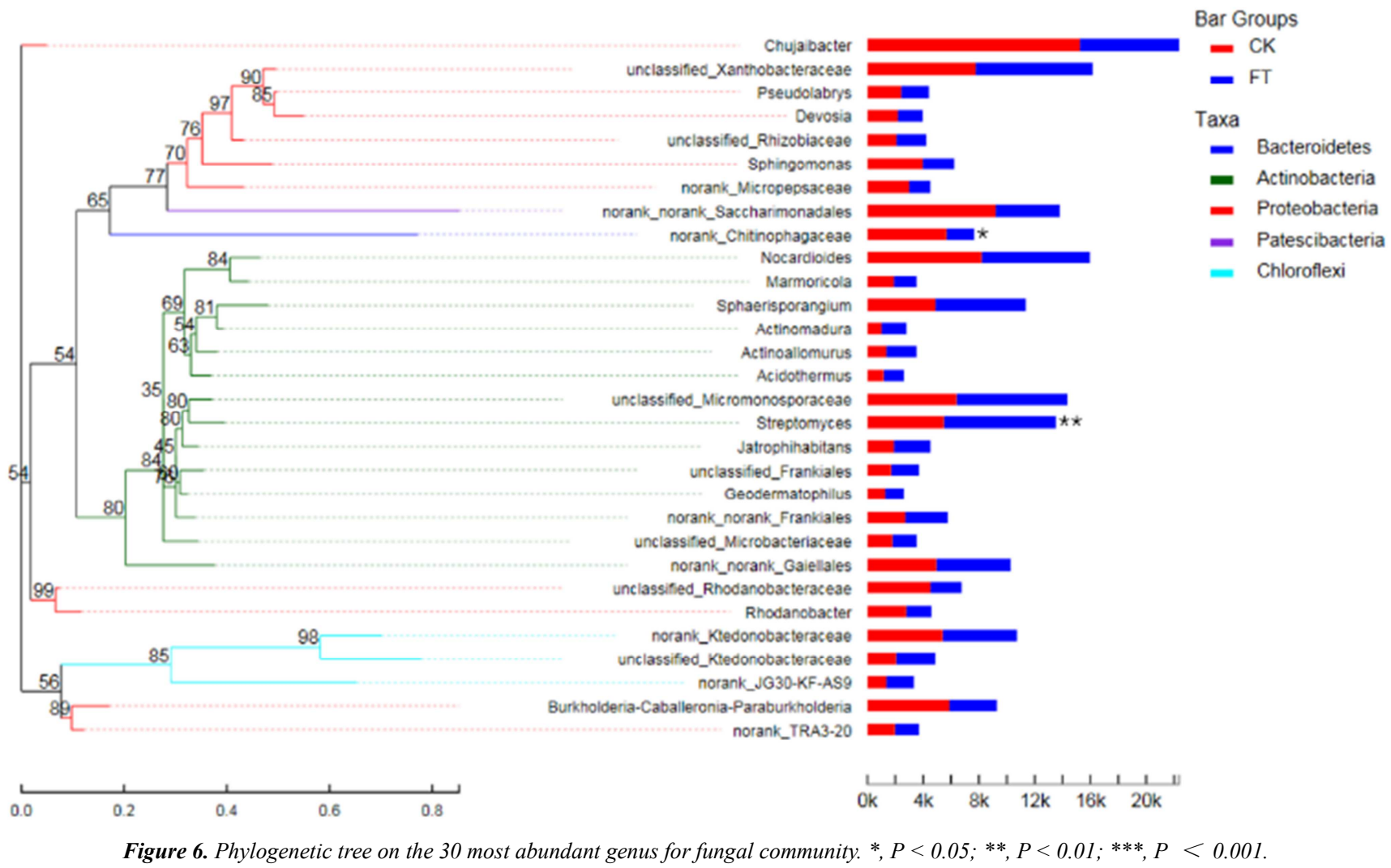

\subsection{Rhizosphere Bacterial Metabolic Profile Prediction}

Using 16S rRNA gene profiling information, PICRUSt [34] predicted the abundance of $\mathrm{C}$ and $\mathrm{N}$ functional genes and found some differences between the rhizosphere soil of FT and the control plants. Analysis of the most abundant N metabolism pathway genes and carbon degrading functional genes revealed that there was a significant decrease in the abundance of $\operatorname{nirK}$ involved in denitrification in the rhizosphere of FT, compared to the control plants $(P=0.047$, Figure 7).

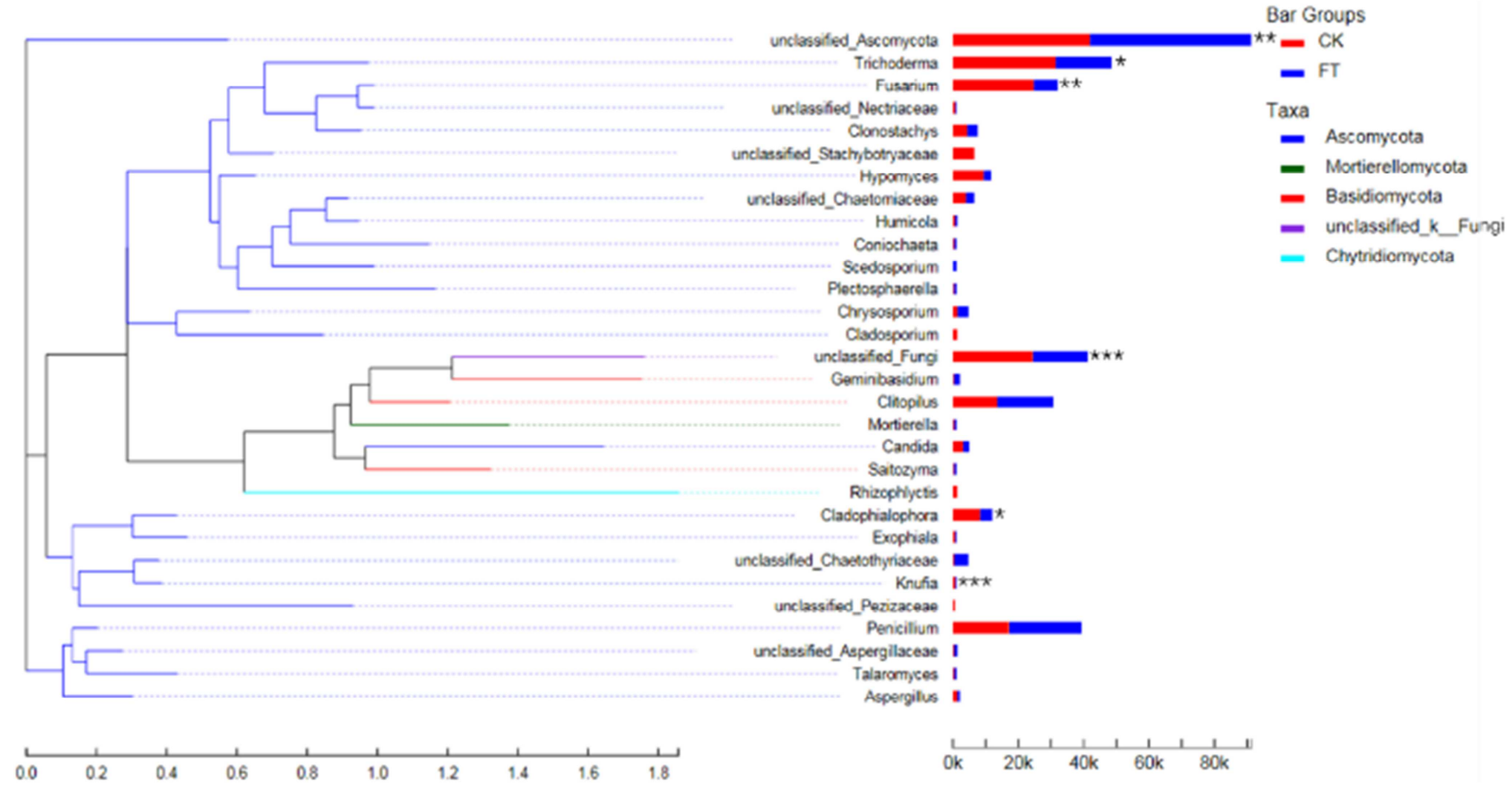

Figure 7. Gene counts of the most abundant PICRUSt predicted $N$ cycling genes (A) and C degrading genes (in order of complexity from labile to recalcitrant) (B). 
Table 5. Correlation coefficients for relationships between soil characteristics and relative abundances of the genus in the rhizosphere of FT and the control plants.

\begin{tabular}{|c|c|c|c|c|c|c|}
\hline & Genus & Electrical conductivity & Organic Carbon & pH & Alkaline nitrogen & Olsen-P \\
\hline \multirow{2}{*}{ Bacteria } & Streptomyces & $-0.878^{*}$ & $-0.814^{*}$ & $-0.599^{\mathrm{a}}$ & $-0.897^{*}$ & $0.175^{\mathrm{a}}$ \\
\hline & Norank Chitinophagaceae & $0.725^{\mathrm{a}}$ & $0.526^{\mathrm{a}}$ & $0.574^{\mathrm{a}}$ & $0.787^{\mathrm{a}}$ & $-0.582^{\mathrm{a}}$ \\
\hline \multirow{6}{*}{ Fungi } & Unclassified Ascomycota & $-0.280^{\mathrm{a}}$ & $-0.017^{\mathrm{a}}$ & $-0.133^{\mathrm{a}}$ & $-0.239^{\mathrm{a}}$ & $0.475^{\mathrm{a}}$ \\
\hline & Trichoderma & $0.743^{\mathrm{a}}$ & $0.655^{\mathrm{a}}$ & $0.696^{\mathrm{a}}$ & $0.893^{*}$ & $-0.589^{\mathrm{a}}$ \\
\hline & Unclassified Fungi & $0.608^{\mathrm{a}}$ & $0.410^{\mathrm{a}}$ & $0.365^{\mathrm{a}}$ & $0.583^{\mathrm{a}}$ & $-0.375^{\mathrm{a}}$ \\
\hline & Fusarium & $0.899^{*}$ & $0.793^{\mathrm{a}}$ & $0.776^{\mathrm{a}}$ & $0.868^{*}$ & $-0.487^{\mathrm{a}}$ \\
\hline & Cladophialophora & $0.852^{*}$ & $0.884^{*}$ & $0.921^{*}$ & $0.841^{*}$ & $-0.460^{\mathrm{a}}$ \\
\hline & Knufia & $0.925^{* *}$ & $0.837^{*}$ & $0.719^{\mathrm{a}}$ & $0.899^{*}$ & $-0.323^{\mathrm{a}}$ \\
\hline
\end{tabular}

Notes: a, not significant; ${ }^{*}, P=0.05$ significance; $* *, P=0.01$ significance.

\section{Discussion}

Surprisingly, after a short term of 45 days' cultivation, the soil in the rhizosphere of Paper mulberry differed significantly in physicochemical properties $(P<0.05)$ compared with those of the initial soil sample, and also the soil properties showed some difference between the two varieties with different nitrogen absorption and utilization efficiency. Correspondingly, soil microbial biomass and microbial composition also exhibited a significant difference between these two varieties. Via the Pearson correlation analysis, we further constructed the relationships between the microbial composition at the genus level and the soil physicochemical properties, and found that alkaline nitrogen was the most significant factor affecting soil microbial community.

Soil nutrients were significantly changed after Paper mulberry cultivation, and the FT plants exhibited stronger nitrogen preference compared to the control plants. Soil physicochemical indexes reduced significantly compared to the initial soil sample, including organic carbon, alkaline nitrogen, and Olsen-P, due to the consumption of Paper mulberry cultivation. Moreover, despite originating from the same soil, the nutrient status of the soil in the rhizosphere of the FT and the control plants differed significantly from each other after 45 days' cultivation (Table 2), especially for alkaline nitrogen and organic carbon. Nitrogen plays a key constituent of nucleic, protein, chlorophyll, alkaloids, vitamins, and hormones [35], is recognized as an essential macronutrient for plants. $\mathrm{N}$ acquisition is closely related to photosynthesis and, therefore, to the yield of crops [36]. In this study, the FT plants acquired a large amount of nitrogen from the soil, and converted to biomacromolecule (e.g. lipoproteins and glycoprotein) [37], exhibited a higher concentration of chlorophyll in leaves, and higher biomass (Table 1). Enhanced nitrogen acquisition from soil strengthened plant photosynthetic capacity and improved dry matter accumulation.

Using high-throughput MiSeq sequencing, the rhizosphere microbiomes of the two Paper mulberry varieties with different nitrogen absorption and utilization efficiency were determined. The $\alpha$-diversity indexes for each sample shown in Table 3 indicated that the FT had a significantly lower fungal diversity (as shown by the lower ACE indices, Shannon index, and Chao1) than the control, however, just
Chaol showed a significant difference for the bacterial community. The principal coordinate analysis (PCoA), the Hierarchical clustering, and taxonomic dissimilarity (Figure 3 and Figure 4) revealed results consistent with those in Table 3, which suggested that the FT and the control plants had significantly different impacts on the composition of the rhizosphere microbiome, and the different impacts appeared more obvious with fungi. Environmental variables (e. g. C/N ratio) that regulate the soil microbiome may firstly affect the fungal composition and have consequences for microbial interactions, which favors soil nutrient cycling [38]. This may explain the different responses of bacteria and fungi to the change of soil physicochemical properties after the short term of the Paper mulberry cultivation (45 days). In the rhizosphere soils, the microbial community turned out to be more similar in the FT plants (Figure 3C and Figure 4C), which was an indication of biotic homogenization [39].

Soil microbial carbon showed significantly lower in the rhizosphere of the FT, compared to the control plants, suggested that soil nitrogen deficiency inhibited soil microbial growth, which was in accordance with the finding that microbiome alleviated nitrogen deficiency by self-feeding under the condition of extreme nitrogen deficiency [40]. The bacterial and fungal community composition at the phylum (Table 4) and the genus levels (Figure 5 and Figure 6) were furtherly determined in the rhizosphere soils of Paper mulberry, and the abundances in the FT and the control plants were compared. The microbial groups differed significantly in the rhizosphere soil of Paper mulberry with different nitrogen absorption and utilization efficiency mainly focused on a few phylum and genus, indicated that the effects of differentiated nitrogen consumption on the soil microbial community showed a strong selectivity, and this is a mode by which host plant nutrient preference shaped special microbiome in the rhizosphere. At the genus level, Streptomyces and norank_Chitinophagaceae from the bacterial community, and Trichoderma, Fusarium, Cladophialophora, and Knufia from the fungal community showed a significant difference between the FT and the control plants, however, the mechanism by which plant roots select specific microbes to assemble in the rhizosphere has not been revealed. Paper mulberry nitrogen preference reduced the microbial growth and changed the bacterial and the fungal composition in the rhizosphere soils. 
Ai et al. [14] suggested that the structure and assembly of the rhizosphere microbiome also depended on other cues, more than the root exudates which were considered key determinants in several studies [13, 41]. However, Ai et al. [14] did not provide further direct evidence in support of this viewpoint. Based on a five-season continuous pot experiment with two plant species, tomato and cucumber, Cai et al. [42] preliminary confirmed that the plant nutrient preference played a key driver in the development of a plant-specific microbiome. The results from the Pearson correlation analysis showed that most of the significant correlations between soil physicochemical properties and microbial genus involved alkaline nitrogen that differed significantly between the two Paper mulberry varieties (Table 5). Also, via function prediction of the bacterial community, nirK involving in nitrogen conversion was found to be lower abundant in the rhizosphere soil of the FT, compared to the control plants (Figure 6). Hence, our study provided further evidence for the role of the nutrient preferences in shaping the rhizosphere microbiome.

\section{Conclusion}

In conclusion, the results obtained from the present study suggested that (i) soil microbial growth and microbial composition significantly differed between the two Paper mulberry varieties with different nitrogen absorption and utilization efficiency; (ii) alkaline nitrogen was identified to be the most significant factor affecting soil microbial community in the rhizosphere soil. Moreover, the effects of excessive consumption of soil nutrient during Paper mulberry cultivation on the microbiome was revealed, and it could be employed in field water and fertilizer management of Paper mulberry planting.

\section{Acknowledgements}

The study was financially supported by the National Natural Science Foundation of China (31960230) and Key Research \& Development projects in Jiangxi Province (2019).

\section{References}

[1] Chaparro, J. M., Badri, D. V., \& Vivanco, J. M., 2013. Rhizosphere microbiome assemblage is affected by plant development. The ISME Journal 8, 790-803.

[2] Brimecombe, M. J., De-Lelj, F. A., Lynch, J. M., The effect of root exudates on rhizosphere microbial populations. In: Pinton, R., Varanini, Z., Nannipieri, P. (eds) The rhizosphere. Biochemistry and organic substances at the soil-plant interface. Marcel Dekker, New York, 2001, 95-140.

[3] Badri, D. V., Vivanco, J. M., 2009. Regulation and function of root exudates. Plant Cell Environment 32, 666-681.

[4] Bakker, M. G., Manter, D. K., Sheflin, A. M., Weir, T. L., Vivanco, J. M., 2012. Harnessing the rhizosphere microbiome through plant breeding and agricultural management. Plant Soil 360, 1-13.

[5] Fakhro, A., Andrade-Linares, D. R., von Bargen, S., Bandte, M., Büttner, C., Grosch, R., Schwarz, D., Franken, P., 2009. Impact of Piriformospora indica on tomato growth and on interaction with fungal and viral pathogens. Mycorrhiza, 20, 191-200.

[6] Youssef, G. H., Seddik, W. M. A., Osman, M. A., 2010. Efficiency of natural minerals in presence of different nitrogen forms and potassium dissolving bacteria on peanut and sesame yields. American Journal of Science 6, 647-660.

[7] Van der Meer, J. R., 1997. Evolution of novel metabolic pathways for the degradation of chloroaromatic compounds. Antonie van Leeuwenhoek 71, 159-178.

[8] Li, T., Hu, Y. J., Hao, Z. P., Li, H., Wang, Y. S., \& Chen, B. D., 2012. First cloning and characterization of two functional aquaporin genes from an arbuscular mycorrhizal fungus Glomus intraradices. New Phytologist 197, 617-630.

[9] Berg, G., Grube, M., Schloter, M., Smalla, K., 2014. Unraveling the plant microbiome: looking back and future perspectives. Frontiers in Microbiology 5, 148.

[10] Mueller, U. G., Sachs, J. L., 2015. Engineering microbiomes to improve plant and animal health. Trends Microbiology 23, 606-617.

[11] Burns, J. H., Anacker, B. L., Strauss, S. Y., \& Burke, D. J., 2015. Soil microbial community variation correlates most strongly with plant species identity, followed by soil chemistry, spatial location and plant genus. AoB PLANTS, 7, plv030-plv030.

[12] Xun, W., Huang, T., Zhao, J., Ran, W., Wang, B., Shen, Q., Zhang, R., 2015. Environmental conditions rather than microbial inoculum composition determine the bacterial composition, microbial biomass and enzymatic activity of reconstructed soil microbial communities. Soil Biology and Biochemistry 90, 10-18.

[13] Ofek, M., Voronov-Goldman, M., Hadar, Y., Minz, D., 2014. Host signature effect on plant root-associated microbiomes revealed through analyses of resident vs. active communities. Environmental Microbiology 16, 2157-2167.

[14] Ai, C., Liang, G., Sun, J., Wang, X., He, P., Zhou, W., He, X., 2015. Reduced dependence of rhizosphere microbiome on plant-derived carbon in 32-year long-term inorganic and organic fertilized soils. Soil Biology and Biochemistry 80, 70-78.

[15] Liao, S. X., Li, K., Yang, Z. Y., Zhang, C. J., 2006. Influence of age on chemical components, fiber morphology and pulping proper- ties of Broussonetia papyrifera bark. Forestry Research 19, 436-440.

[16] Peng, X., Liu, H., Chen, P., Tang, F., Hu, Y., Wang, F., Pi, Z., Zhao, M., Chen, N., Chen, H., Zhang, X., Yan, X., Liu, M., Fu, X., Zhao, G., Yao, P., Wang, L., Dai, H., Li, X., Xiong, W., Xu, W., Zheng, H., Yu, H., and Shen, S., 2019. A Chromosome-Scale Genome Assembly of Paper Mulberry (Broussonetia papyrifera) Provides New Insights into Its Forage and Papermaking Usage. Molecular Plant. 12: 661677.

[17] Zhai, X., Zeng, H., Liu, Y., and Liu, F., 2012. Change of nutrients and shape of Broussonetia papyrifera leaves from different clones. Journal of Northeast Forestry University 40, $38-52$. 
[18] Huang, S., Ding, J., Deng, D., Tang, W., Sun, H., Liu, D., Zhang, L., Niu, X., Zhang, X., Meng, M., et al., 2013. Draft genome of the kiwifruit Actinidia chinensis. Nature Communications. 4, 1-9.

[19] Shen, S., and Peng, X., 2017. The black tech in"Paper Mulberry Poverty Alleviation" fills the lack of crude protein feed material of Chinese animal husbandry. Science, Technology and Development 13, 435-442.

[20] Lichtenthaler, H. K., 1987. Chlorophylls and carotenoids-pigments of photosynthetic biomembranes. Methods in Enzymology 148, 350-382.

[21] Qiu, M., Zhang, R., Xue, C., Zhang, S., Li, S., Zhang, N., and Shen, Q., 2012. Application of bio-organic fertilizer can control Fusarium wilt of cucumber plants by regulating microbial community of rhizosphere soil. Biology and Fertility of Soils 48, 807-816.

[22] Page, A. L., Miller, R. H., Keeney, D. R., 1982. Total carbon, organic carbon and organic matter. In: American Society of Agronomy (Ed.), Methods of Soil Analysis. Part 2, Agronomy. Madison, WI, pp 539-579.

[23] Cornfield, A. H., 1960. Ammonia released on treating soils with $\mathrm{N}$ sodium hydroxide as a possible means of predicting the nitrogen-supplying power of soils. Nature 187, 260-261.

[24] Gyaneshwar, P., Kumar, G. N., Parekh, L. J., and Poole, P. S., 2002. Role of soil microorganisms in improving $P$ nutrition of plants. Food Security in Nutrient-Stressed Environments: Exploiting Plants' Genetic Capabilities. Springer, Netherlands 133-143.

[25] Bergmann, G. T., Bates, S. T., Eilers, K. G., Lauber, C. L., Caporaso, J. G., Walters, W. A., Knight, R., Fierer, N., 2011. The under-recognized dominance of Verrucomicrobia in soil bacterial communities. Soil Biology and Biochemistry 43, 1450-1455.

[26] Rajala, T., Peltoniemi, M., Pennanen, T., and Mäkipää, R., 2012. Fungal community dynamics in relation to substrate quality of decaying Norway spruce (Picea abies [L.] Karst.) logs in boreal forests. FEMS Microbiology Ecology 81, 494-505.

[27] Caporaso, J. G., Kuczynski, J., Stombaugh, J., Bittinger, K., Bushman, F. D., Costello, E. K., Fierer, N., Peña, A. G., Goodrich, J. K., Gordon, J. I., Huttley, G. A., Kelley, S. T., Knights, D., Koenig, J. E., Ley, R. E., Lozupone, C. A., McDonald, D., Muegge, B. D., Pirrung, M., Reeder, J., Sevinsky, J. R., Turnbaugh, P. J., Walters, W. A., Widmann, J., Yatsunenko, T., Zaneveld, J., Knight, R., 2010. QIIME allows analysis of high-throughput community sequencing data. Nature Methods 7, 335-336.

[28] Pruesse, E., Quast, C., Knittel, K., Fuchs, B. M., Ludwig, W., Peplies, J., Glöckner, F. O., 2007. SILVA: a comprehensive online resource for quality checked and aligned ribosomal RNA sequence data compatible with ARB. Nucleic Acids Research 35, 7188-7196.

[29] Hao, X., Jiang, R., Chen, T., 2011. Clustering 16S rRNA for OTU prediction: a method of unsupervised Bayesian clustering. Bioinformatics 27, 611-618.

[30] Henrik-Nilsson, R., Tedersoo, L., Lindahl, B. D., Kjøller, R., Carlsen, T., Quince, C., Abarenkov, K., Pennanen, T., Stenlid,
J., Bruns, T., Larsson, K. H., Kõljalg, U. and Kauserud, H., 2011. Towards standardization of the description and publication of next-generation sequencing datasets of fungal communities. New Phytologist 191, 314-318.

[31] Abarenkov, K., Henrik-Nilsson, R., Larsson, K. H., Alexander, I. J., Eberhardt, U., Erland, S., Høiland, K., Kjøller, R., Larsson, E., Pennanen, T., Sen, R., Taylor, A. F., Tedersoo, L., Ursing, B. M., Vrälstad, T., Liimatainen, K., Peintner, U. and Kõljalg, U., 2010. The UNITE database for molecular identification of fungi-recent updates and future perspectives. New Phytologist 186, 281-285.

[32] Oksanen, J., 2011. Multivariate analysis of ecological communities in R: vegan tutorial. R package version 1 (7).

[33] Clarke, K. R., 1993. Non-parametric multivariate analysis of changes in community structure. Australian Journal of Ecology 18, 117-143.

[34] Langille M. G. I., Zaneveld J., Caporaso J. G., et al., 2013. Predictive functional profiling of microbial communities using 16S rRNA marker gene sequences. Nature biotechnology 31 , 814-821.

[35] Hawkesford, M., Horst, W., Kichey, T., Lambers, H., Schjoerring, J., \& Inge Skrumsager Møller, et al., 2012. Chapter 6 - functions of macronutrients. In Marschners Mineral Nutrition of Higher Plants (3rd ed), pp. 135-189. Academic Press, London.

[36] Wang, Y. Y., Cheng, Y. H., Chen, K. E., et al., 2018. Nitrate transport, signaling, and use efficiency. Annual Review of Plant Biology 69, 85-122.

[37] Wang, G. L., Ding, G. D., Li, L., Cai, H. M., Ye, X. S., Zou, J., \& Xu, F. S., 2014. Identification and characterization of improved nitrogen efficiency in interspecific hybridized new-type Brassica napus. Annals of Botany 114, 549-559.

[38] Bahram, M., Hildebrand, F., Forslund, S. K., Anderson, J. L., Soudzilovskaia, N. A., Bodegom, P. M., Bengtsson-Palme, J., Anslan, S., Coelho, L. P., Harend, H., HUERTA-Cepas, J., Medema, M. H., Maltz, M. R., Mundra, S., Olsson P. A., Pent, M., Põlme, S., Sunagawa, S., Ryberg, M., Tedersoo, L., Bork, P., 2018. Structure and function of the global topsoil microbiome. Nature 560 (7717), 233-237.

[39] Olden, J. D., Poff, N. L., Douglas, M. R., Douglas, M. E., Fausch, K. D., 2004. Ecological and evolutionary consequences of biotic homogenization. Trends in Ecology and Evolution 19, 18-24.

[40] Yu, Z., Peng, Y., Peng, Z., Chai, T., 2013. Influence of nitrogen shortage on activated sludge system. Journal of Harbin University of Commerce 29, 27-31.

[41] Yuan, J., Chaparro, J. M., Manter, D. K., Zhang, R., Vivanco, J. M., Shen, Q., 2015. Roots from distinct plant developmental stages are capable of rapidly selecting their own microbiome without the influence of environmental and soil edaphic factors. Soil Biology and Biochemistry 89, 206-209.

[42] Cai, F., Pang, G., Miao, Y., Li, R., Li, R., Shen, Q., \& Chen, W., 2017. The nutrient preference of plants influences their rhizosphere microbiome. Applied Soil Ecology 110, 146-150. 